\title{
Floristic List for Comanche County, Oklahoma
}

\author{
Bruce Hoagland \\ Oklahoma Biological Survey/Department of Geography \\ University of Oklahoma \\ Norman, OK 73019
}

Floristic lists are vital tools for conservation planning, research, and wildflower appreciation. The diverse physical geography of Comanche County contributes to the high degree of plant diversity and habitats. The county is probably best known for the Wichita Mountains, which are composed of Cambrian gabbro and granites. To the north of the Wichita Mountains is the Slick Hill, an extensive outcropping of limestone. The surface geology of most of the county is composed of Permian sandstones. Land use in the county includes row crop agriculture and pastureland.

\section{INTRODUCTION}

The floristic list presented here was generated form the Atlas of the Flora of Oklahoma database. The database contains records for specimens deposited in Oklahoma herbaria. Voucher specimens for plants on the list can be found at one of the following herbaria: Oklahoma State University, Cameron University, University of Science and Arts of Oklahoma, Southeastern Oklahoma State University, and the Bebb Herbarium at the University of Oklahoma. Taxonomy and common names follow the United States Department of Agricultures Plants Database (www.plants.gov.us).

There are 1,137 species and subspecific taxa, from the 116 families listed. There is a total of 3,560 specimens current recorded in the database. Of those records,
2,009 list the Wichita Mountains as the place of collection and 1,661 list the Wichita Mountains Wildlife Refuge specifically. Other locations in the Wichitas include Medicine Park and Meers. There are 836 records listed for Fort Sill, which includes the Wichita Mountains and a portion of Permian sandstone. The earliest collections in the database are dated 2 July 1903 and were collected by A. Van Vleet in the Wichita Mountains. These specimens are deposited at the Bebb Herbarium. Botanists with large numbers of collections in the database include F. L. Johnson and R. Thompson (790 records for specimens collected as part of florisitic inventory of Fort Sill), F. B. McMurry (498 records, all from the Wichita Mountains National Wildlife Refuge), B. Osborn (262 records), and U.T. Waterfall (121 records).

Hoagland, B.W.

https://doi.org/10.22488/okstate.17.100012 


\section{Floristic List for Comanche County, Oklahoma}

\section{Ferns and allies}

\section{Aspleniaceae}

Asplenium platyneuron (L.) B.S.P. ebony spleenwort

Asplenium resiliens Kunze

blackstem spleenwort

Asplenium trichomanes L.

maidenhair spleenwort

\section{Dryopteridaceae}

Dryopteris marginalis (L.) gray

marginal woodfern

Woodsia obtusa (Spreng.) Torr.

bluntlobe cliff fern

\section{Equisetaceae}

Equisetum hyemale L.

scouringrush horsetail

Equisetum hyemale L.

var. affine (Engelm.) A.A. Eat.

scouringrush horsetail

Equisetum laevigatum A. Braun smooth horsetail

Equisetum $X$ ferrissii Clute (pro sp.)

\section{Marsileaceae}

Marsilea vestita Hook. \& Grev. hairy waterclover

Pilularia americana A. Braun American pillwort

\section{Ophioglossaceae}

Ophioglossum engelmannii Prantl

limestone adderstongue

Ophioglossum vulgatum L. southern adderstongue

\section{Pteridaceae}

Cheilanthes eatonii Baker

Eaton's lipfern

Cheilanthes lanosa (Michx.) D.C. Eat. hairy lipfern

Cheilanthes lindheimeri Hook. fairyswords

Cheilanthes tomentosa Link woolly lipfern
Cheilanthes wootonii Maxon

beaded lipfern

Notholaena sinuata (Lag. ex Sw.)

Kaulfuss

Notholaena standleyi Maxon

star cloak fern

Pellaea atropurpurea (L.) Link purple cliffbrake

Pellaea wrightiana Hook.

Wright's cliffbrake

\section{Selaginellaceae}

Selaginella peruviana (Milde) Hieron.

Peruvian spikemoss

Selaginella rupestris (L.)

Spring

northern selaginella

\section{Gymnosperms}

\section{Cupressaceae}

Juniperus virginiana L. eastern redcedar

Thuja orientalis L. oriental arborvitae

\section{Angiosperms}

\section{Monocotyledonae}

Agavaceae

Manfreda virginica (L.) Salisb. ex

Rose false aloe

Yucca glauca Nutt.

soapweed yucca

Alismataceae

Echinodorus berteroi (Spreng.) Fassett

upright burrhead

Sagittaria brevirostra

Mackenzie \& Bush

Shortbeak arrowhead

Sagittaria calycina Engelm.

hooded arrowhead

Sagittaria calycina Engelm.

var. calycina

hooded arrowhead 
Sagittaria latifolia Willd. broadleaf arrowhead

Araceae

Arisaema dracontium (L.) Schott green dragon

\section{Callitrichaceae}

Callitriche heterophylla Pursh twoheaded water-starwort

\section{Ceratophyllaceae}

Ceratophyllum demersum $\mathrm{L}$. coon's tail

\section{Commelinaceae}

Commelina erecta L. whitemouth dayflower

Commelina erecta L. var. angustifolia (Michx.) Fern. whitemouth dayflower

Commelina erecta L. var. deamiana Fern. whitemouth dayflower

Commelina virginica $L$. Virginia dayflower

Tradescantia ernestiana

E.S. Anderson \& Woods.

Ernest's spiderwort

Tradescantia occidentalis

(Britt.) Smyth

prairie spiderwort

Tradescantia occidentalis (Britt.)

Smyth

var. occidentalis prairie spiderwort

Tradescantia ohiensis

Raf. bluejacket

Tradescantia tharpii

E.S. Anderson \&

Woods.

Tharp's spiderwort
Hydrocharitaceae

Egeria densa Planch.

Brazilian waterweed

\section{Liliaceae}

Allium canadense L. meadow garlic

Allium canadense $\mathrm{L}$. var. fraseri Ownbey

Fraser meadow garlic

Allium canadense L. var. hyacinthoides (Bush) Ownbey \& Aase hyacinth meadow garlic

Allium canadense $\mathrm{L}$. var. mobilense (Regel) Ownbey meadow garlic

Allium drummondii Regel Drummond's onion

Allium stellatum Nutt. ex Ker-Gawl. autumn onion

Androstephium coeruleum (Scheele) Greene [orthographic variant]

Camassia angusta

(Engelm. \& Gray) Blank. prairie camas

Camassia scilloides

(Raf.) Cory

Atlantic camas

Cooperia drummondii Herbert evening rainlily

Erythronium albidum

Nutt.

white fawnlily

Erythronium

mesochoreum Knerr

midland fawnlily 
Erythronium rostratum

W. Wolf

yellow troutlily

Nolina texana S. Wats.

Texas sacahuista

Nothoscordum bivalve

(L.) Britt.

crowpoison

Polygonatum biflorum

(Walt.) Ell.

smooth Solomon's

seal

Polygonatum biflorum

(Walt.) Ell.

var. commutatum

(J.A. \& J.H. Schultes)

Morong smooth

Solomon's seal

Zigadenus nuttallii (Gray)

S. Wats.

Nuttall's deathcamas

\section{Najadaceae}

Najas guadalupensis (Spreng.) Magnus southern waternymph

\section{Orchidaceae}

Spiranthes cernua (L.)

L.C. Rich.

nodding

ladies'-tresses

Spiranthes

magnicamporum

Sheviak

Great Plains

ladies'-tresses

Spiranthes vernalis Engelm. \& Gray

spring ladies'-tresses

\section{Poaceae}

Aegilops cylindrica Host

jointed goatgrass

Agropyron smithii Rydb.

Agrostis hyemalis (Walt.)

B.S.P.

winter bentgrass
Alopecurus carolinianus

Walt.

Carolina foxtail

Andropogon gerardii

Vitman

big bluestem

Aristida curtissii

(Gray ex S. Wats. \&

Coult.) Nash

Aristida dichotoma

Michx.

churchmouse

threeawn

Aristida dichotoma Michx.

var. curtissii

Gray ex S. Wats. \& Coult.

Curtis' threeawn

Aristida longispica Poir.

var. longispica

slimspike threeawn

Aristida oligantha Michx.

prairie threeawn

Aristida purpurascens

Poir.

arrowfeather

threeawn

Aristida purpurea Nutt.

var. fendleriana

(Steud.) Vasey

Fendler's threeawn

Aristida purpurea Nutt.

var. longiseta (Steud.)

Vasey

Fendler threeawn

Bothriochloa barbinodis

(Lag.) Herter

cane bluestem

Bothriochloa ischaemum

(L.) Keng

yellow bluestem

Bothriochloa

saccharoides

(Sw.) Rydb.

silver bluestem

Bouteloua gracilis

(Willd. ex Kunth) Lag. 
ex Griffiths

blue grama

Bouteloua hirsuta Lag.

hairy grama

Bouteloua pectinata

Featherly

Bouteloua rigidiseta

(Steud.)

A.S. Hitchc.

Texas grama

Bromus catharticus Vahl

rescuegrass

Bromus commutatus

Schrad.

meadow brome

Bromus japonicus Thunb.

ex Murr.

Japanese brome

Bromus pubescens Muhl. ex Willd.

hairy woodland brome

Bromus tectorum L.

cheatgrass

Buchloe dactyloides

(Nutt.) Engelm.

buffalograss

Cenchrus incertus M.A.

Curtis

Cenchrus longispinus

(Hack.) Fern.

mat sandbur

Chasmanthium latifolium

(Michx.) Yates

Indian woodoats

Chloris verticillata Nutt.

tumble windmill grass

Chloris virgata Sw. feather fingergrass

Cinna arundinacea L. sweet woodreed

Cynodon dactylon (L.)

Pers.

Bermudagrass

Dactylis glomerata L.

orchardgrass
Dichanthelium acuminatum (Sw.)

Gould \& C.A. Clark

tapered rosette grass

Dichanthelium acuminatum (Sw.)

Gould \& C.A. Clark

var. fasciculatum

(Torr.) Freckmann

western panicgrass

Dichanthelium acuminatum (Sw.)

Gould \& C.A. Clark

var. lindheimeri

(Nash) Gould \& C.A. Clark

Lindheimer panicgrass

Dichanthelium linearifolium

(Scribn. ex Nash) Gould

slimleaf panicgrass

Dichanthelium malacophyllum

(Nash) Gould

softleaf rosette grass

Dichanthelium oligosanthes

(J.A. Schultes) Gould Heller's

rosette grass

Dichanthelium oligosanthes

(J.A. Schultes) Gould

var. scribnerianum (Nash) Gould

Scribner's rosette grass

Digitaria californica (Benth.) Henr.

Arizona cottontop

Digitaria cognata

(J.A. Schultes) Pilger

Carolina crabgrass

Digitaria ischaemum (Schreb.)

Schreb. ex Muhl.

smooth crabgrass

Digitaria sanguinalis (L.) Scop.

hairy crabgrass

Echinochloa colona (L.) Link

jungle rice

Echinochloa crus-galli (L.) Beauv. barnyardgrass

Echinochloa crus-galli (L.) Beauv. barnyardgrass

Eleusine indica (L.) Gaertn. Indian goosegrass

Eragrostis barrelieri Daveau

Mediterranean lovegrass

Hoagland, B.W. 
Eragrostis capillaris (L.) Nees lace grass

Eragrostis cilianensis (All.) Vign. ex Janchen stinkgrass

Eragrostis curtipedicellata Buckl. gummy lovegrass

Eragrostis curvula (Schrad.) Nees weeping lovegrass

Eragrostis hypnoides (Lam.) B.S.P. teal lovegrass

Eragrostis intermedia A.S. Hitchc. plains lovegrass

Eragrostis pectinacea (Michx.) Nees ex Steud. tufted lovegrass

Eragrostis pectinacea (Michx.) Nees ex Steud. var. pectinacea tufted lovegrass

Eragrostis pilosa (L.) Beauv. Indian lovegrass

Eragrostis reptans (Michx.) Nees

Eragrostis secundiflora J. Presl red lovegrass

Eragrostis sessilispica Buckl. tumble lovegrass

Eragrostis spectabilis (Pursh) Steud. purple lovegrass

Eragrostis trichodes (Nutt.) Wood sand lovegrass

Eriochloa contracta A.S. Hitchc. prairie cupgrass

Eriochloa sericea (Scheele) Munro ex Vasey Texas cupgrass

Erioneuron pilosum (Buckl.) Nash hairy woollygrass

Festuca octoflora Walt.

Festuca subverticillata

(Pers.) Alexeev

nodding fescue

Leersia oryzoides (L.) Sw. rice cutgrass

Leersia virginica Willd. whitegrass

Leptochloa fascicularis (Lam.) Gray
Leptochloa mucronata (Michx.) Kunth

Melica nitens (Scribn.) Nutt. ex Piper threeflower melicgrass

Muhlenbergia racemosa (Michx.) B.S.P. marsh muhly

Muhlenbergia sobolifera (Muhl. ex Willd.) Trin. rock muhly

Panicum anceps Michx beaked panicgrass

Panicum capillare L. witchgrass

Panicum dichotomiflorum Michx. fall panicgrass

Panicum hallii Vasey Hall's panicgrass

Panicum hillmanii Chase Hillman's panicgrass

Panicum obtusum Kunth vine mesquite

Panicum virgatum L. switchgrass

Pascopyrum smithii (Rydb.) A. Löve western wheatgrass

Paspalidium geminatum (Forsk.) Stapf Egyptian panicgrass

Paspalum distichum L. knotgrass

Paspalum pubiflorum Rupr. ex Fourn. hairyseed paspalum

Paspalum pubiflorum Rupr. ex Fourn. var. glabrum Vasey ex Scribn.

Paspalum setaceum Michx. thin paspalum

Paspalum setaceum Michx. var. stramineum (Nash) D. Banks

Phalaris caroliniana Walt. Carolina canarygrass

Poa annua L. annual bluegrass 
Poa arachnifera Torr.

Texas bluegrass

Poa compressa L.

Canada bluegrass

Schedonnardus paniculatus

(Nutt.) Trel.

tumblegrass

Schizachyrium scoparium

(Michx.) Nash

little bluestem

Setaria glauca (L.) Beauv.

Sphenopholis obtusata (Michx.)

Scribn.

prairie wedgescale

Sporobolus airoides (Torr.) Torr.

alkali sacaton

Sporobolus asper (Michx.) Kunth

Sporobolus asper (Michx.) Kunth

var. drummondii (Trin.) Vasey

Sporobolus clandestinus (Biehler)

A.S. Hitchc.

rough dropseed

Sporobolus cryptandrus (Torr.) Gray

sand dropseed

Sporobolus neglectus Nash

puffsheath dropseed

Sporobolus ozarkanus Fern.

Sporobolus pyramidatus (Lam.)

A.S. Hitchc.

Sporobolus vaginiflorus

(Torr. ex Gray) Wood

poverty dropseed

Tripsacum dactyloides (L.) L. eastern gamagrass

Vulpia octoflora (Walt.) Rydb. sixweeks fescue

\section{Potamogetonaceae}

Potamogeton amplifolius Tuckerman largeleaf pondweed

Potamogeton crispus L. curly pondweed

Potamogeton diversifolius Raf. waterthread pondweed

Potamogeton illinoensis Morong Illinois pondweed
Potamogeton nodosus Poir. longleaf pondweed

Potamogeton pusillus L. small pondweed

Potamogeton pusillus L. ssp. pusillus small pondweed

Potamogeton pusillus L. var. tenuissimus Mert. \& Koch

Typhaceae

Typha angustifolia L. narrowleaf cattail

Typha domingensis Pers. southern cattail

Typha latifolia L. broadleaf cattail

\section{Zannichelliaceae}

Zannichellia palustris L. horned pondweed

\section{Acanthaceae}

\section{Dicotyledonae}

Dicliptera brachiata (Pursh) Spreng. branched foldwing Justicia americana (L.) Vahl American water-willow

Ruellia ciliosa Pursh.

Ruellia humilis Nutt. fringeleaf wild petunia

Ruellia humilis Nutt. var. expansa Fern.

Ruellia humilis Nutt. var. longiflora (Gray) Fern.

\section{Aceraceae}

Acer negundo L.

boxelder

Acer negundo L. var. interius (Britt.) Sarg. boxelder

Acer negundo L. var. texanum Pax boxelder

Acer saccharinum L. silver maple 
Acer saccharum Marsh. sugar maple

\section{Amaranthaceae}

Amaranthus albus L. prostrate pigweed

Amaranthus arenicola I.M. Johnston sandhill amaranth

Amaranthus graecizans L.

Amaranthus hybridus L. slim amaranth

Amaranthus retroflexus L. redroot amaranth

Amaranthus rudis Sauer tall amaranth

Froelichia floridana (Nutt.) Moq. plains snakecotton

Froelichia gracilis (Hook.) Moq. slender snakecotton

Gossypianthus lanuginosus (Poir.) Moq. woolly cottonflower

Gossypianthus lanuginosus (Poir.) Moq. var. lanuginosus woolly cottonflower

Iresine rhizomatosa Standl. Juda's bush

\section{Anacardiaceae}

Rhus aromatica Ait. fragrant sumac

Rhus aromatica Ait. var. serotina (Greene) Rehd. fragrant sumac

Rhus glabra L. smooth sumac

Rhus trilobata Nutt. var. trilobata skunkbush sumac

Toxicodendron radicans (L.) Kuntze eastern poison ivy

\section{Apiaceae}

Ammoselinum popei Torr. \& Gray plains sandparsley

Chaerophyllum procumbens

(L.) Crantz spreading chervil
Chaerophyllum tainturieri Hook. hairyfruit chervil

Chaerophyllum tainturieri Hook. var. dasycarpum Hook. ex S. Wats.

Chaerophyllum tainturieri Hook. var. tainturieri

Chaerophyllum texanum Coult. \& Rose

Cicuta maculata L. spotted water hemlock

Conium maculatum $\mathrm{L}$. poison hemlock

Daucus carota L.

Queen Anne's lace

Daucus pusillus Michx. American wild carrot

Eryngium hookerii Walp. Hooker's eryngo

Eryngium leavenworthii Torr. \& Gray Leavenworth's eryngo

Limnosciadium pinnatum (DC.) Mathias \& Constance tansy dogshade

Lomatium daucifolium

(Torr. \& Gray) Coult. \& Rose

Lomatium foeniculaceum (Nutt.)

Coult. \& Rose ssp. daucifolium

(Torr. \& Gray) Theobald desert biscuitroot

Polytaenia nuttallii DC. Nutall's prairie parsley

Sanicula canadensis L.

Canadian blacksnakeroot

Spermolepis divaricata

(Walt.) Raf. ex Ser.

roughfruit scaleseed

Spermolepis echinata

(Nutt. ex DC.) Heller

bristly scaleseed

Spermolepis inermis (Nutt. ex DC.)

Mathias \& Constance

Red River scaleseed

Spermolepis patens

(Nutt. ex DC.) B.L. Robins. 
Torilis nodosa (L.) Gaertn. knotted hedgeparsley

\section{Apocynaceae}

Amsonia ciliata Walt. fringed bluestar

Amsonia ciliata Walt. var. texana (Gray) Coult.

Texas bluestar

Amsonia illustris Woods.

Ozark bluestar

Apocynum cannabinum $\mathrm{L}$. Indianhemp

Apocynum cannabinum L. var. glaberrimum A. DC.

\section{Asclepiadaceae}

Asclepias asperula (Dcne.) Woods. spider milkweed

Asclepias asperula (Dcne.) Woods. ssp. capricornu (Woods.) Woods. antelopehorns

Asclepias asperula (Dcne.) Woods. var. decumbens (Nutt.) Shinners Asclepias engelmanniana Woods. Engelmann's milkweed

Asclepias pumila (Gray) Vail plains milkweed

Asclepias stenophylla Gray slimleaf milkweed

Asclepias sullivantii Engelm. ex Gray prairie milkweed

Asclepias tuberosa L. butterfly milkweed

Asclepias tuberosa L. ssp. interior Woods. butterfly milkweed

Asclepias verticillata $\mathrm{L}$. whorled milkweed

Asclepias viridiflora Raf. green comet milkweed

Ascleias viridis Walt. green antelopehorn Asclepiodora decumbens (Nutt.) Gray
Matelea biflora (Raf.) Woods. star milkvine

Asteraceae

Achillea millefolium $\mathrm{L}$. common yarrow

Achillea millefolium $\mathrm{L}$. var. occidentalis DC. western yarrow

Ambrosia artemisiifolia L. annual ragweed

Ambrosia artemisiifolia L. var. elatior (L.) Descourtils annual ragweed

Ambrosia psilostachya DC.

Cuman ragweed

Ambrosia trifida L. great ragweed

Ambrosia trifida L. var. texana Scheele Texan great ragweed

Amphiachyris dracunculoides (DC.) Nutt. prairie broomweed Antennaria parlinii Fern. ssp. fallax (Greene) Bayer \& Stebbins Parlin's pussytoes Antennaria plantaginifolia (L.)

Richards.

woman's tobacco

Aphanostephus pilosus Buckl.

hairy dozedaisy

Aphanostephus skirrhobasis

(DC.) Trel.

Arkansas dozedaisy

Artemisia carruthii Wood ex Carruth.

Carruth's sagewort

Artemisia dracunculus L. tarragon

Artemisia filifolia Torr. sand sagebrush

Artemisia ludoviciana Nutt. white sagebrush 
Artemisia ludoviciana Nutt. ssp. Iudoviciana white sagebrush

Artemisia ludoviciana Nutt. ssp. mexicana (Willd. ex Spreng.) Keck white sagebrush

Artemisia neomexicana Greene ex Rydb.

Aster ericoides $\mathrm{L}$.

Aster oblongifolius Nutt.

Aster oblongifolius Nutt. var. rigidulus Gray Aster ontarionis Wieg.

Aster patens Ait.

Aster patens Ait. var. patentissimus (Lindl. ex DC.) Torr. \& Gray

Aster subulatus Michx.

Aster subulatus Michx. var. ligulatus Shinners Astranthium integrifolium (Michx.) Nutt. ssp. ciliatum (Raf.) DeJong entireleaf western daisy

Baccharis salicina Torr. \& Gray Great Plains false willow

Bidens bipinnata L. Spanish needles

Bidens frondosa $\mathrm{L}$. devil's beggartick

Brickellia eupatorioides (L.) Shinners var. chlorolepis (Woot. \& Standl.) B.L. Turner false boneset

Brickellia eupatorioides (L.) Shinners var. corymbulosa (Torr. \& Gray) Shinners false boneset

Centaurea americana Nutt. American star-thistle Chaetopappa asteroides Nutt. ex DC.

Arkansas leastdaisy Chrysopsis berlandieri Greene Chrysopsis pilosa Nutt. soft goldenaster
Chrysopsis stenophylla (Gray) Greene

Chrysopsis villosa (Pursh) Nutt. ex DC.

Chrysopsis villosa (Pursh) Nutt. ex DC. var. stenophylla (Gray) Gray

Cirsium altissimum (L.) Hill tall thistle

Cirsium ochrocentrum Gray yellowspine thistle

Cirsium texanum Buckl.

Texas thistle

Cirsium undulatum (Nutt.) Spreng. wavyleaf thistle

Cirsium undulatum (Nutt.) Spreng. var. megacephalum (Gray) Fern.

Conyza canadensis (L.) Cronq. Canadian horseweed

Conyza canadensis (L.) Cronq. var. canadensis Canadian horseweed

Conyza ramosissima Cronq. dwarf horseweed

Coreopsis grandiflora Hogg ex Sweet largeflower tickseed

Coreopsis grandiflora Hogg ex Sweet var. harveyana (Gray) Sherff largeflower tickseed

Coreopsis grandiflora Hogg ex Sweet var. longipes (Hook.) Torr. \& Gray largeflower tickseed

Coreopsis tinctoria Nutt. golden tickseed

Coreopsis tinctoria Nutt. var. tinctoria golden tickseed

Dracopis amplexicaulis (Vahl) Cass. clasping coneflower

Dysodiopsis tagetoides

(Torr. \& Gray) Rydb. false dogfennel 
Dyssodia tagetoides Torr. \& Gray

Echinacea angustifolia DC.

blacksamson echinacea

Echinacea purpurea (L.) Moench eastern purple coneflower

Eclipta prostrata (L.) L. false daisy

Elephantopus carolinianus Raeusch.

Carolina elephantsfoot

Engelmannia pinnatifida Gray ex Nutt.

Erigeron canadensis L.

Erigeron philadelphicus L.

Philadelphia fleabane

Erigeron ramosus (Walt.) B.S.P.

Erigeron strigosus Muhl. ex Willd. prairie fleabane

Erigeron strigosus Muhl. ex Willd.

var. strigosus

prairie fleabane

Erigeron tenuis Torr. \& Gray slenderleaf fleabane

Evax prolifera Nutt. ex DC.

bighead pygmycudweed

Filago prolifera (Nutt. ex DC.) Britt., non Pomel

Gaillardia lanceolata Michx.

Gaillardia lutea Greene

Gaillardia pulchella Foug. firewheel

Gaillardia suavis (Gray \& Engelm.)

Britt. \& Rusby

perfumeballs

Gnaphalium obtusifolium L.

Gnaphalium wrightii Gray

Grindelia nuda Wood var. nuda curlytop gumweed

Grindelia squarrosa (Pursh) Dunal curlycup gumweed

Grindelia squarrosa (Pursh) Dunal

var. nuda (Wood) Gray

Gutierrezia dracunculoides

(DC.) Blake

Gutierrezia sarothrae (Pursh)

Britt. \& Rusby

broom snakeweed
Gutierrezia texana (DC.) Torr. \&

Gray

var. texana

Texas snakeweed

Haplopappus ciliatus (Nutt.) DC.

Haplopappus divaricatus (Nutt.)

Gray

Haplopappus spinulosus (Pursh)

DC.

Haplopappus spinulosus (Pursh)

DC.

ssp. australis (Greene) Hall

Haplopappus spinulosus (Pursh)

DC.

ssp. cotulus (Small) Hall

Haplopappus spinulosus (Pursh)

DC.

ssp. glaberrimus (Rydb.) Hall

Haplopappus spinulosus (Pursh)

DC.

var. turbinellus (Rydb.) Blake

Helenium amarum (Raf.) H. Rock yellowdicks

Helenium amarum (Raf.) H. Rock

var. badium

(Gray ex S. Wats.) Waterfall

yellowdicks

Helenium badium (Gray ex S. Wats.)

Greene

Helenium microcephalum DC. smallhead sneezeweed

Helianthus annuus L.

common sunflower

Helianthus hirsutus Raf.

hairy sunflower

Helianthus maximiliani Schrad.

Maximilian sunflower

Helianthus orgyalis DC.

Helianthus petiolaris Nutt.

prairie sunflower

Helianthus salicifolius A. Dietr.

willowleaf sunflower

Heterotheca canescens

(DC.) Shinners

hoary false goldenaster

Heterotheca latifolia Buckl. 
Heterotheca latifolia Buckl. var. macgregoris Wagenkn.

Heterotheca stenophylla (Gray) Shinners stiffleaf false goldenaster

Heterotheca villosa (Pursh) Shinners hairy false goldenaster

Heterotheca villosa var. villosa (Pursh) Shinners hairy goldenaster

Hieracium longipilum Torr. hairy hawkweed

Hymenopappus corymbosus

Torr. \& Gray

Hymenopappus scabiosaeus L'Hér. Carolina woollywhite

Hymenopappus scabiosaeus L'Hér. var. corymbosus (Torr. \& Gray)

B.L. Turner

Carolina woollywhite

Hymenopappus tenuifolius Pursh

Chalk Hill hymenopappus

Hymenoxys linearifolia Hook.

Iva annua L.

annual marshelder

Iva annua L. var. annua annual marshelder

Krigia biflora (Walt.) Blake twoflower dwarfdandelion

Krigia caespitosa (Raf.) Chambers weedy dwarfdandelion

Krigia cespitosa (Raf.) Chambers [orthographic variant]

Krigia dandelion (L.) Nutt. potato dwarfdandelion

Krigia occidentalis Nutt. western dwarfdandelion

Kuhnia eupatorioides L. false boneset

Kuhnia eupatorioides L. var. corymbulosa Torr. \& Gray Kuhnia glutosina Ell. false boneset

Lactuca canadensis L. Canada lettuce
Lactuca canadensis L. var. latifolia Kuntze

Lactuca Iudoviciana (Nutt.) Riddell biannual lettuce

Lactuca scariola L. var. integrata Gren. \& Godr.

Lactuca serriola L. prickly lettuce

Liatris aspera Michx. tall blazing star

Liatris punctata Hook. dotted blazing star

Liatris punctata Hook. var. nebraskana Gaiser Nebraska blazing star

Liatris scabra (Greene) K. Schum.

Liatris squarrosa (L.) Michx. scaly blazing star

Lindheimera texana Gray \& Engelm.

Texas yellowstar

Machaeranthera annua

(Rydb.) Shinners

Machaeranthera spinulosa (Greene) Cory hoary tansyaster

Packera plattensis (Nutt.) W.A. Weber \& A. Löve prairie groundsel

Packera tampicana (DC.) C. Jeffrey Great Plains ragwort

Palafoxia rosea (Bush) Cory rosy palafox

Palafoxia rosea (Bush) Cory var. rosea rosy palafox

Pluchea camphorata (L.) DC. camphor pluchea

Pluchea odorata (L.) Cass. var. odorata sweetscent

Prenanthes altissima L. tall rattlesnakeroot

Prionopsis ciliata (Nutt.) Nutt. Pyrrhopappus grandiflorus

(Nutt.) Nutt.

tuberous desert-chicory 
Rudbeckia hirta L. blackeyed Susan

Rudbeckia hirta L. var. pulcherrima Farw. blackeyed Susan

Rudbeckia hirta L. var. serotina (Nutt.) Core

Rudbeckia serotina Nutt.

Senecio plattensis Nutt.

Silphium asperrimum Hook.

Silphium asteriscus L. starry rosinweed

Silphium laciniatum $\mathrm{L}$. compassplant

Solidago arguta Ait. Atlantic goldenrod

Solidago canadensis L. var. gilvocanescens Rydb. shorthair goldenrod

Solidago delicatula Small

Solidago gigantea Ait. giant goldenrod

Solidago missouriensis Nutt. Missouri goldenrod

Solidago missouriensis Nutt. var. fasciculata Holz. Missouri goldenrod

Solidago mollis Bartl. velvety goldenrod

Solidago nemoralis Ait. gray goldenrod

Solidago nemoralis Ait. ssp. longipetiolata (Mackenzie \& Bush) G.W. Douglas

Solidago petiolaris Ait. downy ragged goldenrod

Solidago petiolaris Ait. var. petiolaris downy ragged goldenrod

Solidago rigida $\mathrm{L}$.

Solidago speciosa Nutt. var. rigidiuscula Torr. \& Gray showy goldenrod
Solidago ulmifolia Muhl. ex Willd. var. microphylla Gray elmleaf goldenrod

Sonchus asper (L.) Hill spiny sowthistle

Taraxacum officinale G.H. Weber ex Wiggers common dandelion

Tetraneuris acaulis (Pursh) Greene stemless four-nerve daisy

Tetraneuris linearifolia (Hook.) Greene fineleaf fournerved daisy

Thelesperma filifolium (Hook.) Gray stiff greenthread

Thelesperma filifolium (Hook.) Gray var. filifolium stiff greenthread

Thelesperma intermedium Rydb. Thelesperma trifidum (Poir.) Britt.

Townsendia exscapa (Richards.) Porter stemless Townsend daisy

Tragopogon dubius Scop. yellow salsify

Verbesina alternifolia (L.)

Britt. ex Kearney wingstem

Verbesina encelioides (Cav.) Benth. \& Hook. f. ex Gray ssp. encelioides golden crownbeard Verbesina virginica $\mathrm{L}$. white crownbeard Vernonia baldwinii Torr. Baldwin's ironweed Vernonia baldwinii Torr. ssp. interior (Small) Faust interior ironweed Vernonia interior Small Vernonia missurica Raf. Missouri ironweed Xanthisma texanum DC. Texas sleepydaisy Xanthisma texanum DC. ssp. drummondii 
(Torr. \& Gray) Semple Drummond's sleepydaisy

Xanthisma texanum DC.

var. drummondii

(Torr. \& Gray) Gray

Xanthium strumarium L. rough cockleburr

Xanthium strumarium L. var. canadense (P. Mill.)

Torr. \& Gray

Canada Cocklebur

\section{Bignoniaceae}

Campsis radicans (L.) Seem.

ex Bureau

trumpet creeper

Catalpa bignonioides Walt. southern catalpa

Catalpa speciosa (Warder) Warder ex Engelm.

northern catalpa

\section{Boraginaceae}

Heliotropium tenellum (Nutt.) Torr. pasture heliotrope

Lithospermum incisum Lehm. narrowleaf stoneseed

Myosotis verna Nutt.

spring forget-me-not

Onosmodium molle Michx. ssp. occidentale (Mackenzie) Cochrane western marbleseed

\section{Brassicaceae}

Arabis fendleri (S. Wats.) Greene Fendler's rockcress

Arabis laevigata (Muhl. ex Willd.) Poir. smooth rockcress

Arabis missouriensis Greene green rockcress

Camelina microcarpa DC. littlepod false flax

Capsella bursa-pastoris (L.) Medik. shepherd's purse
Chorispora tenella (Pallas) DC crossflower

Descurainia pinnata (Walt.) Britt. ssp. brachycarpa (Richards.) Detling western tansymustard

Draba brachycarpa Nutt. ex Torr. \& Gray shortpod draba

Draba cuneifolia Nutt. ex Torr. \& Gray wedgeleaf draba

Draba cuneifolia Nutt. ex Torr. \& Gray var. cuneifolia wedgeleaf draba

Draba cuneifolia Nutt. ex Torr. \& Gray var. helleri (Small) O.E. Schulz

Draba platycarpa Torr. \& Gray broadpod draba

Draba reptans (Lam.) Fern. Carolina draba

Erysimum asperum (Nutt.) DC.

Erysimum asperum (Nutt.) DC. var. arkansanum (Nutt.) Gray

Erysimum capitatum (Dougl. ex Hook.) Greene sanddune wallflower

Erysimum capitatum

(Dougl. ex Hook.)

Greene var. capitatum sanddune wallflower

Erysimum repandum $\mathrm{L}$. spreading wallflower

Lepidium austrinum Small southern pepperwort Lepidium densiflorum Schrad. common pepperweed

Lepidium densiflorum Schrad. var. densiflorum common pepperweed Lepidium oblongum Small veiny pepperweed Lepidium virginicum $\mathrm{L}$. Virginia pepperweed 
Lepidium virginicum L.

var. medium (Greene) C.L.

Hitchc.

medium pepperweed

Lesquerella auriculata

(Engelm. \& Gray) S. Wats. earleaf bladderpod

Lesquerella gordonii (Gray) S. Wats. Gordon's bladderpod

Lesquerella gracilis (Hook.) S. Wats. spreading bladderpod

Lesquerella gracilis (Hook.) S. Wats. ssp. nuttallii

(Torr. \& Gray) Rollins \& Shaw

Nuttall's bladderpod

Lesquerella ovalifolia Rydb.

ex Britt.

roundleaf bladderpod

Lesquerella ovalifolia Rydb. ex Britt. ssp. alba (Goodman)

Rollins \& Shaw

roundleaf bladderpod

Nasturtium officinale Ait. f.

Rorippa nasturtium-aquaticum (L.)

Hayek

watercress

Rorippa palustris (L.) Bess.

bog yellowcress

Rorippa sessiliflora (Nutt.)

A.S.Hitchc.

stalkless yellowcress

Sibara virginica (L.) RollinsVirginia winged rockcress

\section{Cactaceae}

Echinocereus baileyi Rose

Echinocereus reichenbachii

(Terscheck ex Walp.) Haage f.

lace hedgehog cactus

Echinocereus reichenbachii

(Terscheck ex Walp.) Haage f.

var. albispinus (Lahman)

L. Benson

Echinocereus reichenbachii

(Terscheck ex Walp.) Haage f. var. baileyi (Rose) N.P. Taylor

Bailey's hedgehog cactus

Opuntia engelmannii Salm-Dyck

cactus apple

Opuntia humifusa (Raf.) Raf.

devil's-tongue

\section{Campanulaceae}

Lobelia appendiculata $A$. DC. pale lobelia

Lobelia cardinalis $L$.

cardinalflower

Lobelia cardinalis L.

ssp. graminea (Lam.) McVaugh

Triodanis leptocarpa (Nutt.) Nieuwl. slimpod Venus' looking-glass

Triodanis perfoliata (L.) Nieuwl. var. biflora (Ruiz \& Pavón)

Bradley

clasping Venus' looking-glass

\section{Capparaceae}

Cleome serrulata Pursh

Rocky Mountain beeplant

Cleomella angustifolia Torr. narrowleaf rhombopod

Polanisia dodecandra (L.) DC. redwhisker clammyweed

Polanisia dodecandra (L.) DC.

ssp.trachysperma

(Torr. \& Gray) Iltis

sandyseed clammyweed

\section{Caprifoliaceae}

Lonicera albiflora Torr. \& Gray

western

white honeysuckle

Lonicera japonica Thunb.

Japanese honeysuckle

Sambucus nigra L. ssp. Canadensis

(L.) R. Bolli

common elderberry

Symphoricarpos orbiculatus Moench

coralberry

Viburnum rufidulum Raf.

rusty blackhaw

Hoagland, B.W. 


\section{Caryophyllaceae}

Cerastium brachypodum

(Engelm. ex Gray) B.L. Robins. shortstalk chickweed

Cerastium glomeratum Thuill. sticky chickweed

Minuartia michauxii (Fenzl) Farw. Michaux's stitchwort

Minuartia michauxii (Fenzl) Farw. var. texana (B.L. Robins.) Mattf. Texas stitchwort

Minuartia patula (Michx.) Mattf. pitcher's stitchwort

Minuartia patula var. patula (Michx.) Mattf.

Paronychia jamesii Torr. \& Gray James' nailwort

Paronychia virginica Spreng. yellow nailwort

Sagina decumbens (Ell.) Torr. \& Gray trailing pearlwort

Silene antirrhina $\mathrm{L}$. sleepy silene

Celastraceae Celastrus scandens $\mathrm{L}$. American bittersweet

Euonymus atropurpurea Jacq. eastern wahoo

\section{Chenopodiaceae}

Chenopodium album L. lambsquarters

Chenopodium ambrosioides $\mathrm{L}$. Mexican tea

Chenopodium leptophyllum (Moq.) Nutt. ex S. Wats. narrowleaf goosefoot

Chenopodium pratericola Rydb. desert goosefoot

Chenopodium simplex (Torr.) Raf. mapleleaf goosefoot

Chenopodium standleyanum Aellen Standley's goosefoot
Kochia scoparia (L.) Schrad. Mexican-fireweed

Salsola kali L. prickly Russian thistle

Salsola kali L. ssp. tragus (L.) Celak.

\section{Cistaceae}

Lechea tenuifolia Michx. narrowleaf pinweed

Clusiaceae

Hypericum drummondii (Grev. \& Hook.)

Torr. \& Gray nits and lice

Hypericum mutilum L. dwarf St. Johnswort

\section{Convolvulaceae}

Convolvulus arvensis L. field bindweed

Convolvulus equitans Benth.

Texas bindweed

Convolvulus incanus auct. non Vahl

Evolvulus nuttallianus J.A. Schultes shaggy dwarf morning-glory

Evolvulus pilosus Nutt. Ipomoea pandurata (L.) G.F.W. Mey.

man of the earth

Ipomoea shumardiana

(Torr.) Shinners

narrowleaf morning-glory

\section{Cornaceae}

Cornus drummondii C.A. Mey. roughleaf dogwood

\section{Crassulaceae}

Sedum nuttallianum Raf. yellow stonecrop

\section{Cucurbitaceae}

Cucurbita foetidissima Kunth

Missouri gourd

Cyclanthera dissecta

(Torr. \& Gray) Arn.

cutleaf cyclanthera 
Ibervillea lindheimeri (Gray) Greene

Lindheimer's globeberry

Melothria pendula L.

Guadeloupe cucumber

Cuscutaceae

Cuscuta glabrior (Engelm.) Yuncker var. pubescens (Engelm.)

Yuncker

Cuscuta gronovii Willd. ex J.A.

Schultes

scaldweed

Cuscuta indecora Choisy

bigseed alfalfa dodder

Cuscuta indecora Choisy

var. indecora

bigseed alfalfa dodder

Cuscuta pentagona Engelm.

fiveangled dodder

Cuscuta pentagona Engelm.

var. pentagona

fiveangled dodder

Cyperaceae

Bolboschoenus maritimus (L.) Palla

Bulbostylis capillaris (L.)

Kunth ex C.B. Clarke

densetuft hairsedge

Bulbostylis capillaris (L.) Kunth

ex C.B. Clarke ssp. capillaries

densetuft hairsedge

Carex albicans Willd. ex Spreng.

var. albicans

whitetinge sedge

Carex austrina Mackenzie southern sedge

Carex blanda Dewey eastern woodland sedge

Carex brevior (Dewey) Mackenzie shortbeak sedge

Carex emoryi Dewey

Emory's sedge

Carex fissa Mackenzie hammock sedge

Carex frankii Kunth Frank's sedge

Carex granularis Muhl. ex Willd. limestone meadow sedge
Carex gravida Bailey heavy sedge

Carex gravida Bailey var. Iunelliana (Mackenzie) F.J. Herm. heavy sedge

Carex grisea Wahlenb.

Carex joorii Bailey cypress swamp sedge

Carex muehlenbergii Schkuhr ex Willd. var. enervis Boott Muhlenberg's sedge

Carex muehlenbergii Schkuhr ex Willd. var. muehlenbergii Muhlenberg's sedge

Carex tetrastachya Scheele Britton's sedge

Carex umbellata Schkukr ex Willd. parasol sedge

Carex vulpinoidea Michx. fox sedge

Cyperus acuminatus Torr. \& Hook. ex Torr. tapertip flatsedge

Cyperus echinatus (L.) Wood globe flatsedge

Cyperus erythrorhizos Muhl. redroot flatsedge

Cyperus esculentus L.

chufa flatsedge

Cyperus ferax L.C. Rich.

Cyperus filiculmis auct. non Vahl

Cyperus hallii Britt.

Cyperus Iupulinus (Spreng.) Marcks

ssp. Iupulinus

Great Plains flatsedge

Cyperus odoratus L.

fragrant flatsedge

Cyperus ovularis (Michx.) Torr.

Cyperus pseudovegetus Steud.

marsh flatsedge

Cyperus schweinitzii Torr.

Schweinitz's flatsedge

Cyperus setigerus Torr. \& Hook.

lean flatsedge

Cyperus squarrosus L.

bearded flatsedge

Hoagland, B.W. 
Cyperus strigosus L. strawcolored flatsedge

Eleocharis acutisquamata Buckl. sharpscale spikerush

Eleocharis atropurpurea (Retz.) J. \&. K. Presl. purple spikerush

Eleocharis compressa Sullivant flatstem spikerush

Eleocharis engelmannii Steud. Engelmann's spikerush

Eleocharis erythropoda Steud. bald spikerush

Eleocharis montevidensis Kunth sand spikerush

Eleocharis obtusa (Willd.) J.A. Schultes blunt spikerush

Eleocharis palustris (L.) Roemer \& J.A. Schultes common spikerush

Eleocharis parvula

(Roemer \& J.A. Schultes) Link ex Bluff, Nees \& Schauer dwarf spikerush

Eleocharis quadrangulata (Michx.)

Roemer \& J.A. Schultes squarestem spikerush

Fimbristylis puberula (Michx.) Vahl hairy fimbry

Fimbristylis puberula (Michx.) Vahl var. puberula hairy fimbry

Fimbristylis spadicea auct. non (L.) Vahl.

Fimbristylis vahlii (Lam.) Link Vahl's fimbry

Fuirena simplex Vahl western umbrella-sedge Hemicarpha aristulata (Coville) Smyth

Hemicarpha drummondii Nees Hemicarpha micrantha (Vahl) Pax Lipocarpha aristulata (Coville)

G. Tucker awned halfchaff sedge
Lipocarpha drummondii (Nees)

G. Tucker

Drummond's halfchaff sedge

Lipocarpha micrantha (Vahl)

G. Tucker

smallflower halfchaff sedge

Schoenoplectus acutus (Muhl. ex Bigelow) A.\& D. Löve var. acutus hardstem bulrush

Schoenoplectus americanus (Pers.) Volk. ex Schinz \& R. Keller chairmaker's bulrush

Schoenoplectus hallii (Gray) S.G.

Sm.

Hall's bulrush

Schoenoplectus pungens (Vahl)

Palla

common threesquare

Schoenoplectus tabernaemontani

(K.C. Gmel.) Palla

softstem bulrush

Scirpus americanus Pers.

Scirpus atrovirens Willd.

green bulrush

Scirpus lineatus Michx.

rusty bulrush, drooping bulrush

Scirpus pallidus (Britt.) Fern.

cloaked bulrush

Scirpus pendulus Muhl.

rufous bulrush

Scleria ciliata Michx.

fringed nutrush

Scleria pauciflora Muhl. ex Willd.

fewflower nutrush

Ebenaceae

Diospyros virginiana L.

common persimmon

\section{Elatinaceae}

Bergia texana (Hook.) Seub. ex

Walp.

Texas bergia 


\section{Euphorbiaceae}

Acalypha gracilens Gray var. monococca Engelm. ex Gray

Acalypha monococca

(Engelm. ex Gray) L.

Mill. \&Gandhi

slender threeseed mercury

Acalypha ostryifolia Riddell

pineland threeseed mercury

Acalypha virginica $\mathrm{L}$.

Virginia threeseed mercury

Argythamnia mercurialina

(Nutt.) Muell.-Arg.

tall silverbush

Argythamnia mercurialina (Nutt.)

Muell.-Arg. var. mercurialina

tall silverbush

Chamaesyce maculata (L.) Small

spotted sandmat

Chamaesyce missurica

(Raf.) Shinners

prairie sandmat

Chamaesyce nutans (Lag.) Small

eyebane

Chamaesyce prostrata (Ait.) Small prostrate sandmat

Chamaesyce serpens (Kunth) Small matted sandmat

Cnidoscolus texanus

(Muell.-Arg.) Small

Texas bullnettle

Croton capitatus Michx.

hogwort

Croton capitatus Michx. var.

capitatus

hogwort

Croton capitatus Michx.

var. lindheimeri

(Engelm. \& Gray) Muell.-Arg.

Lindheimer's hogwort

Croton glandulosus L.

vente conmigo

Croton glandulosus L.

var. septentrionalis Muell.-Arg.

vente conmigo
Croton lindheimerianus Scheele threeseed croton

Croton monanthogynus Michx. prairie tea

Croton texensis (Klotzsch)

Muell.-Arg.

Texas croton

Euphorbia chamaesyce auct. non L.

Euphorbia corollata L.

flowering spurge

Euphorbia cyathophora Murr. fire on the mountain

Euphorbia dentata Michx. toothed spurge

Euphorbia lata Engelm.

Euphorbia longicruris Scheele wedgeleaf spurge

Euphorbia maculata L.

Euphorbia marginata Pursh snow on the mountain

Euphorbia missurica Raf.

Euphorbia nutans Lag.

Euphorbia serpens Kunth

Euphorbia spathulata Lam.

warty spurge

Euphorbia supina Raf.

Phyllanthus abnormis Baill.

Drummond's leaf-flower

Phyllanthus abnormis Baill.

var. abnormis

Drummond's leaf-flower

Phyllanthus caroliniensis Walt.

Carolina leaf-flower

Phyllanthus polygonoides

Nutt. ex Spreng.

smartweed leaf-flower

Stillingia sylvatica Garden ex L.

queen's-delight

Tragia ramosa Torr.

branched noseburn

Fabaceae

Acacia angustissima (P. Mill.)

Kuntze

var. hirta (Nutt.) B.L. Robins.

prairie acacia

Hoagland, B.W. 
Amorpha canescens Pursh leadplant

Amorpha fruticosa $\mathrm{L}$. desert false indigo

Apios americana Medik. groundnut

Astragalus caryocarpus Ker-Gawl.

Astragalus crassicarpus

Nutt. var. crassicarpus

groundplum milkvetch

Astragalus crassicarpus Nutt. var. trichocalyx (Nutt.) Barneby groundplum milkvetch

Astragalus distortus Torr. \& Gray Ozark milkvetch

Astragalus lindheimeri Engelm. ex Gray

Lindheimer's milkvetch

Astragalus nuttallianus DC. var. nuttallianus smallflowered milkvetch

Astragalus plattensis Nutt. Platte River milkvetch

Baptisia australis (L.) R. Br. ex Ait. f. blue wild indigo

Baptisia australis (L.) R. Br. ex Ait. f. var. minor (Lehm.) Fern. blue wild indigo

Baptisia bracteata Muhl. ex Ell. longbract wild indigo

Baptisia bracteata Muhl. ex Ell. var. glabrescens (Larisey) Isely

Baptisia bracteata Muhl. ex Ell. var. leucophaea (Nutt.) Kartesz \& Gandhi longbract wild indigo

Baptisia leucophaea Nutt. Baptisia sphaerocarpa Nutt. yellow wild indigo

Baptisia Vent. wild indigo

Cassia fasciculata Michx. Cassia marilandica L.

Cercis canadensis L. eastern redbud
Chamaecrista fasciculata

(Michx.) Greene sleepingplant

Clitoria mariana L. Atlantic pigeonwings

Crotalaria sagittalis L. arrowhead rattlebox

Dalea aurea Nutt. ex Pursh golden prairie clover

Dalea candida Michx. ex Willd. white prairie clover

Dalea candida Michx. ex Willd. var. candida white prairie clover

Dalea candida Michx. ex Willd. var. oligophylla (Torr.) Shinners white prairie clover

Dalea enneandra Nutt. nineanther prairie clover

Dalea frutescens Gray black prairie clover

Dalea multiflora (Nutt.) Shinners roundhead prairie clover

Dalea purpurea Vent. violet prairie clover

Dalea purpurea Vent. var. purpurea violet prairie clover

Dalea tenuifolia (Gray) Shinners slimleaf prairie clover

Dalea villosa (Nutt.) Spreng. var. villosa silky prairie clover

Desmanthus illinoensis (Michx.) MacM. ex B.L. Robins. \& Fern. prairie bundleflower

Desmanthus leptolobus Torr. \& Gray slenderlobe bundleflower

Desmodium canescens (L.) DC. hoary ticktrefoil

Desmodium ciliare

(Muhl. ex Willd.) DC.

hairy small-leaf ticktrefoil

Desmodium dillenii Darl. p.p.

Desmodium glutinosum

(Muhl. ex Willd.) Wood

pointedleaf ticktrefoil 
Desmodium illinoense Gray

Illinois ticktrefoil

Desmodium nudiflorum (L.) DC. nakedflower ticktrefoil

Desmodium paniculatum (L.) DC. panicledleaf ticktrefoil

Desmodium paniculatum (L.) DC. var. paniculatum panicledleaf ticktrefoil

Desmodium sessilifolium (Torr.)

Torr. \& Gray sessileleaf ticktrefoil

Desmodium tweedyi Britt. Tweedy's ticktrefoil

Desmodium viridiflorum (L.) DC. velvetleaf ticktrefoil

Galactia volubilis (L.) Britt. downy milkpea

Gleditsia triacanthos L. honeylocust

Gymnocladus dioicus (L.) K. Koch Kentucky coffeetree

Hoffmannseggia glauca (Ortega) Eifert Indian rushpea

Hosackia americana (Nutt.) Piper Indigofera miniata Ortega coastal indigo

Indigofera miniata Ortega var. leptosepala

(Nutt. ex Torr. \& Gray) B.L.

Turner western indigo

Lespedeza capitata Michx. roundhead lespedeza

Lespedeza intermedia sensu Clewell, 1966

Lespedeza procumbens Michx. trailing lespedeza

Lespedeza repens (L.) W. Bart. creeping lespedeza

Lespedeza virginica (L.) Britt. slender lespedeza

Lotus purshianus

F. E. \& E. S. Clements
Lotus unifoliolatus (Hook.) Benth. var. unifoliolatus

American bird's-foot trefoil

Lupinus texensis Hook.

Texas lupine

Medicago minima (L.) L.

Bur medick

Medicago sativa L. alfalfa

Melilotus albus

Melilotus officinalis (L.) Lam. yellow sweetclover

Mimosa nuttallii (DC.) B.L. Turner Nuttall's sensitive-briar

Neptunia lutea (Leavenworth) Benth. yellow puff

Oxytropis lambertii Pursh purple locoweed

Oxytropis lambertii Pursh var. articulata (Greene) Barneby purple locoweed

Oxytropis lambertii Pursh var. lambertii purple locoweed

Pediomelum cuspidatum (Pursh) Rydb. largebract Indian breadroot

Pediomelum esculentum (Pursh) Rydb. large Indian breadroot

Pediomelum linearifolium (Torr. \& Gray) J. Grimes narrowleaf Indian breadroot

Petalostemon occidentalis (Heller) Fern.

Petalostemon purpureus

(Vent.) Rydb.

Prosopis glandulosa Torr. honey mesquite

Prosopis glandulosa Torr. var. glandulosa honey mesquite Prosopis juliflora (Sw.) DC. var. glandulosa (Torr.) Cockerell Psoralea argophylla Pursh

Hoagland, B.W. 
Psoralea cuspidata Pursh

Psoralea esculenta Pursh

Psoralea linearifolia Torr. \& Gray

Psoralea tenuiflora Pursh

Psoralidium lanceolatum

(Pursh) Rydb.

lemon scurfpea

Psoralidium tenuiflorum

(Pursh) Rydb.

slimflower scurfpea

Robinia pseudoacacia L.

black locust

Schrankia uncinata Willd.

Senna marilandica (L.) Link

Maryland senna

Sophora affinis Torr. \& Gray

Eve's necklacepod

Strophostyles helvula (L.) Ell.

trailing fuzzybean

Strophostyles leiosperma

(Torr. \& Gray) Piper

slickseed fuzzybean

Tephrosia onobrychoides Nutt. multibloom hoarypea

Tephrosia virginiana (L.) Pers. Virginia tephrosia

Trifolium dubium Sibthorp suckling clover

Trifolium reflexum $\mathrm{L}$. buffalo clover

Trifolium repens $\mathrm{L}$. white clover

Vicia americana Muhl. ex Willd. ssp. minor (Hook.) C.R. Gunn mat vetch

Vicia caroliniana Walt.

Carolina vetch

Vicia ludoviciana Nutt. Louisiana vetch

Vicia ludoviciana Nutt. ssp. Iudoviciana Louisiana vetch

Vicia ludoviciana Nutt. var. texana (Torr. \& Gray) Shinners

Vicia texana (Torr. \& Gray) Small
Fagaceae

Quercus buckleyi Nixon \& Dorr

Buckley oak

Quercus fusiformis Small plateau oak

Quercus macrocarpa Michx. bur oak

Quercus marilandica Muench. blackjack oak

Quercus mohriana Buckl. ex Rydb. Mohr oak

Quercus muehlenbergii Engelm. chinkapin oak

Quercus shumardii Buckl. Shumard's oak

Quercus stellata Wangenh. post oak

Quercus velutina Lam. black oak

\section{Fumariaceae}

Corydalis aurea Willd. scrambled eggs

Corydalis aurea Willd. ssp. occidentalis (Engelm. ex Gray) G.B. Ownbey Corydalis curvisiliqua Engelm. curvepod fumewort

Corydalis curvisiliqua Engelm. ssp. grandibracteata (Fedde) G.B. Ownbey curvepod fumewort

Corydalis micrantha (Engelm. ex Gray) Gray smallflower fumewort

Corydalis micrantha (Engelm. ex Gray) Gray ssp. micrantha smallflower fumewort

\section{Gentianaceae}

Centaurium texense (Griseb.) Fern. Lady Bird's centaury Eustoma russellianum (Hook.) G. Don 
Sabatia campestris Nutt.

Texas star

Geraniaceae

Erodium cicutarium (L.) L'Hér. ex Ait. redstem stork's bill

Geranium carolinianum L.

Carolina geranium

\section{Grossulariaceae}

Ribes aureum Pursh

var. villosum DC.

golden currant

\section{Haloragaceae}

Myriophyllum aquaticum (Vell.)

Verdc.

parrot feather watermilfoil

Myriophyllum heterophyllum Michx. twoleaf watermilfoil

Myriophyllum pinnatum (Walt.)

B.S.P.

cutleaf watermilfoil

Myriophyllum sibiricum Komarov

shortspike watermilfoil

Myriophyllum spicatum L.

spike watermilfoil

Myriophyllum spicatum L.

var. exalbescens (Fern.) Jepson

\section{Hippocastanaceae}

Aesculus glabra Willd.

Ohio buckeye

Aesculus glabra Willd. var. arguta

(Buckl.) B.L. Robins.

Ohio buckeye

\section{Hydrophyllaceae}

Ellisia nyctelea (L.) L.

Aunt Lucy

Nama hispidum Gray

bristly nama

Nemophila phacelioides Nutt.

largeflower baby blue eyes

Phacelia congesta Hook.

caterpillars
Phacelia strictiflora

(Engelm. \& Gray) Gray

prairie phacelia

Phacelia strictiflora (Engelm. \&

Gray)

Gray var. lundelliana Constance

Lundell's phacelia

\section{Iridaceae}

Nemastylis geminiflora Nutt.

prairie pleatleaf

Sisyrinchium angustifolium P. Mill. narrowleaf blue-eyed grass

Sisyrinchium campestre Bickn.

prairie blue-eyed grass

\section{Isoetaceae}

Isoetes melanopoda Gay \& Durieu

ex Durieu

blackfoot quillwort

Juglandaceae

Carya cordiformis

(Wangenh.) K. Koch

bitternut hickory

Carya illinoinensis

(Wangenh.) K. Koch

pecan

Juglans microcarpa Berl.

little walnut

Juglans nigra L.

black walnut

Juncaceae

Juncus acuminatus Michx. tapertip rush

Juncus aristulatus Michx.

Juncus brachycarpus Engelm.

whiteroot rush

Juncus diffusissimus Buckl.

slimpod rush

Juncus dudleyi Wieg.

Dudley's rush

Juncus interior Wieg.

inland rush

Juncus marginatus Rostk.

grassleaf rush 
Juncus marginatus Rostk. var. setosus Coville

Juncus nodatus Coville stout rush

Juncus scirpoides Lam. needlepod rush

Juncus secundus Beauv. ex Poir. lopsided rush

Juncus tenuis Willd. poverty rush

Juncus torreyi Coville Torrey's rush

\section{Krameriaceae}

Krameria lanceolata Torr. trailing krameria

\section{Lamiaceae}

Hedeoma hispida Pursh rough false pennyroyal Hedeoma reverchonii (Gray) Gray Reverchon's false pennyroyal Lamium amplexicaule L. henbit deadnettle

Lycopus americanus Muhl. ex W. Bart.

American water horehound

Lycopus americanus Muhl. ex W. Bart. var. scabrifolius Fern.

Mentha spicata L. spearmint

Monarda citriodora Cerv. ex Lag. lemon beebalm

Monarda clinopodioides Gray basil beebalm

Monarda dispersa Small

Monarda fistulosa L. wild bergamot

Monarda mollis L.

Monarda pectinata Nutt. pony beebalm Monarda punctata L. spotted beebalm Nepeta cataria L. catnip
Prunella vulgaris L. common selfheal

Salvia azurea Michx. ex Lam. azure blue sage

Scutellaria drummondii Benth. Drummond's skullcap

Scutellaria ovata Hill heartleaf skullcap

Scutellaria wrightii Gray Wright's skullcap

Teucrium canadense L. Canada germander

Teucrium canadense L. var. occidentale (Gray) McClintock \& Epling western germander

Teucrium canadense $\mathrm{L}$. var. virginicum (L.) Eat.

Teucrium laciniatum Torr. lacy germander

\section{Lentibulariaceae}

Utricularia gibba L. humped bladderwort

\section{Linaceae}

Linum berlandieri Hook. var. berlandieri Berlandier's yellow flax Linum hudsonioides Planch.

Texas flax Linum imbricatum (Raf.) Shinners tufted flax

Linum lewisii Pursh prairie flax

Linum pratense (J.B.S. Norton)

Small

meadow flax

Linum rigidum Pursh stiffstem flax

Linum rigidum Pursh var. berlandieri (Hook.)

Torr. \& Gray

Linum rigidum Pursh var. rigidum stiffstem flax 
Linum sulcatum Riddell grooved flax

Linum sulcatum Riddell var. sulcatum grooved flax

\section{Loasaceae}

Mentzelia albescens

(Gillies \& Arn.) Griseb.

wavyleaf blazingstar

Mentzelia decapetala

(Pursh ex Sims)

Urban \& Gilg ex Gilg

tenpetal blazingstar

Mentzelia oligosperma Nutt. ex Sims chickenthief

Mentzelia reverchonii (Urban \& Gilg)

H.J. Thompson \& Zavortink

Reverchon's blazingstar

\section{Lythraceae}

Ammannia auriculata Willd. eared redstem

Ammannia coccinea Rottb. valley redstem

Ammannia robusta Heer \& Regel grand redstem

Lythrum alatum Pursh winged lythrum

Lythrum alatum Pursh var. lanceolatum (EII.)

Torr. \& Gray ex Rothrock

Rotala ramosior (L.) Koehne lowland rotala

\section{Malvaceae}

Abutilon incanum (Link) Sweet pelotazo

Callirhoe alcaeoides (Michx.) Gray light poppymallow

Callirhoe digitata Nutt. winecup

Callirhoe involucrata

(Torr. \& Gray) Gray

purple poppymallow
Callirhoe involucrata (Torr. \& Gray) Gray var. involucrata purple poppymallow

Callirhoe leiocarpa R.F. Martin tall poppymallow

Callirhoe pedata

(Nutt. ex Hook.) Gray

palmleaf poppymallow

Hibiscus trionum $\mathrm{L}$. flower of an hour

Sida spinosa L. prickly fanpetals

Sphaeralcea coccinea (Nutt.) Rydb. scarlet globemallow

Sphaeralcea coccinea (Nutt.)Rydb. ssp. coccinea

scarlet globemallow

Menispermaceae

Cocculus carolinus (L.) DC.

Carolina coralbead

Menispermum canadense L. common moonseed

\section{Molluginaceae}

Mollugo verticillata $\mathrm{L}$. green carpetweed

Moraceae

Maclura pomifera (Raf.) Schneid. osage orange

Morus alba L. white mulberry

Morus rubra L. red mulberry

Nelumbonaceae

Nelumbo lutea Willd. American lotus

Nyctaginaceae

Mirabilis albida (Walt.) Heimerl white four o'clock

Mirabilis glabra (S. Wats.) Standl. smooth four o'clock

Mirabilis hirsuta (Pursh) MacM. hairy four o'clock 
Mirabilis linearis (Pursh) Heimerl narrowleaf four o'clock

Mirabilis nyctaginea (Michx.) MacM. heartleaf four o'clock

\section{Nymphaeaceae}

Nymphaea odorata Ait.

American white waterlily

\section{Oleaceae}

Fraxinus pennsylvanica Marsh. green ash

\section{Onagraceae}

Calylophus berlandieri Spach Berlandier's sundrops

Calylophus berlandieri Spach ssp. pinifolius

(Engelm. ex Gray) Towner Berlandier's sundrops

Calylophus hartwegii (Benth.) Raven ssp. pubescens (Gray)

Towner \& Raven Hartweg's sundrops

Calylophus serrulatus (Nutt.) Raven yellow sundrops

Gaura coccinea Nutt. ex Pursh scarlet beeblossom

Gaura longiflora Spach longflower beeblossom

Gaura parviflora Dougl. ex Lehm.

Gaura sinuata Nutt. ex Ser. wavyleaf beeblossom

Gaura suffulta Engelm. ex Gray kisses

Gaura suffulta Engelm. ex Gray ssp. suffulta kisses

Gaura triangulata Buckl. prairie beeblossom

Jussiaea diffusa auct. non Forsk.

Jussiaea repens L. var. glabrescens Kuntze

Ludwigia alternifolia L. seedbox

Ludwigia decurrens Walt. wingleaf primrose-willow
Ludwigia palustris (L.) Ell. marsh seedbox

Ludwigia peploides (Kunth) Raven ssp. glabrescens (Kunze) Raven floating primrose-willow

Ludwigia repens J.R. Forst. creeping primrose-willow

Oenothera biennis L. common evening-primrose

Oenothera grandis (Britt.) Smyth showy evening-primrose

Oenothera laciniata Hill cutleaf evening-primrose

Oenothera linifolia Nutt. threadleaf evening-primrose

Oenothera macrocarpa Nutt. ssp. incana (Gray) Wagner bigfruit evening-primrose

Oenothera macrocarpa Nutt. ssp. macrocarpa bigfruit evening-primrose

Oenothera speciosa Nutt. pinkladies

Oenothera triloba Nutt. stemless evening-primrose

Stenosiphon linifolius

(Nutt. ex James) Heynh. false gaura

Oxalidaceae

Oxalis corniculata L. creeping woodsorrel

Oxalis dillenii Jacq.

Oxalis stricta L. common yellow oxalis

Oxalis violacea $\mathrm{L}$. violet woodsorrel

\section{Papaveraceae}

Argemone polyanthemos

(Fedde) G.B. Ownbey crested prickly poppy

Papaver dubium L.

blindeyes 
Passifloraceae

Passiflora lutea L.

yellow passionflower

\section{Pedaliaceae}

Proboscidea louisianica

(P. Mill.) Thellung

ram's horn

Phytolaccaceae

Phytolacca americana L. American pokeweed

Rivina humilis L. rougeplant

\section{Plantaginaceae}

Plantago aristata Michx. largebracted plantain

Plantago elongata Pursh prairie plantain

Plantago major L. common plantain

Plantago patagonica Jacq. woolly plantain

Plantago pusilla Nutt. dwarf plantain

Plantago rhodosperma Dcne. redseed plantain

Plantago rugelii Dcne. blackseed plantain

Plantago virginica L. Virginia plantain

Plantago wrightiana Dcne. Wright's plantain

\section{Platanaceae}

Platanus occidentalis L. American sycamore

\section{Polemoniaceae}

Gilia aggregata (Pursh) Spreng. ssp. formosissima (Greene) Wherry

Gilia rubra (L.) Heller Ipomopsis rubra (L.) Wherry standing-cypress
Phlox longipilosa Waterfall longhair phlox

\section{Polygalaceae}

Polygala alba Nutt. white milkwort

Polygala verticillata L. whorled milkwort

Polygala verticillata $\mathrm{L}$. var. isocycla Fern. whorled milkwort

\section{Polygonaceae}

Eriogonum annuum Nutt. annual buckwheat

Eriogonum longifolium Nutt. longleaf buckwheat

Eriogonum longifolium Nutt. var. longifolium longleaf buckwheat

Persicaria punctata (Ell.) Small

Polygonum amphibium L. var. emersum Michx. longroot smartweed

Polygonum aviculare L. prostrate knotweed

Polygonum bicorne Raf.

Polygonum convolvulus L. black bindweed

Polygonum hydropiper L. marshpepper knotweed

Polygonum hydropiperoides Michx. swamp smartweed

Polygonum lapathifolium L. curlytop knotweed

Polygonum pensylvanicum L. Pennsylvania smartweed

Polygonum persicaria L. spotted ladysthumb

Polygonum punctatum Ell. dotted smartweed

Polygonum ramosissimum Michx. bushy knotweed

Polygonum ramosissimum Michx. var. prolificum Small bushy knotweed 
Polygonum scandens L. climbing false buckwheat

Polygonum striatulum B.L. Robins. striped knotweed

Polygonum tenue Michx. pleatleaf knotweed

Polygonum virginianum $\mathrm{L}$. jumpseed

Rumex altissimus Wood pale dock

Rumex crispus L. curly dock

\section{Pontederiaceae}

Heteranthera limosa (Sw.) Willd.

blue mudplantain

Pontederia cordata L. pickerelweed

\section{Portulacaceae}

Claytonia virginica L. Virginia springbeauty

Portulaca pilosa L. kiss me quick

Portulaca umbraticola Kunth ssp. lanceolata (Engelm.) Matthews \& Ketron

Talinum calycinum Engelm. largeflower fameflower

Talinum parviflorum Nutt. sunbright

\section{Primulaceae}

Androsace occidentalis Pursh western rockjasmine

Dodecatheon meadia L. pride of Ohio

Dodecatheon meadia L. ssp. meadia pride of Ohio

Samolus floribundus Kunth

Samolus parviflorus Raf.

Samolus valerandi L. ssp. parviflorus

(Raf.) Hultén

seaside brookweed
Ranunculaceae

Anemone berlandieri Pritz. tenpetal thimbleweed

Anemone caroliniana Walt.

Carolina anemone

Anemone decapetala auct. non Ard.

Clematis pitcheri Torr. \& Gray bluebill

Clematis versicolor Small ex Rydb. pale leather flower

Consolida ajacis (L.) Schur doubtful knight's-spur

Delphinium carolinianum Walt. Carolina larkspur

Delphinium carolinianum Walt. ssp. virescens (Nutt.) Brooks

Carolina larkspur

Myosurus minimus L. tiny mousetail

Ranunculus abortivus L. littleleaf buttercup

Ranunculus longirostris Godr. longbeak buttercup

Ranunculus sceleratus $\mathrm{L}$. cursed buttercup

\section{Rhamnaceae}

Ceanothus herbaceus Raf. Jersey tea

\section{Rosaceae}

Agrimonia parviflora Ait. harvestlice

Crataegus crus-galli L. cockspur hawthorn

Crataegus engelmannii Sarg. Engelmann's hawthorn

Crataegus mollis Scheele Arnold hawthorn

Crataegus reverchonii Sarg. Reverchon's hawthorn

Crataegus viridis L. green hawthorn

Crataegus viridis L. var. viridis green hawthorn 
Fragaria virginiana Duchesne Virginia strawberry

Geum canadense Jacq. white avens

Geum canadense Jacq. var. camporum

(Rydb.) Fern. \& Weatherby white avens

Potentilla arguta Pursh tall cinquefoil

Potentilla arguta Pursh ssp. arguta tall cinquefoil

Prunus angustifolia Marsh. Chickasaw plum

Prunus gracilis Engelm. \& Gray Oklahoma plum

Prunus hortulana Bailey hortulan plum

Prunus mexicana S. Wats. Mexican plum

Prunus persica (L.) Batsch peach

Prunus rivularis Scheele creek plum

Prunus virginiana $\mathrm{L}$. chokecherry

Rosa foliolosa Nutt. ex Torr. \& Gray white prairie rose

Rosa multiflora Thunb. ex Murr. multiflora rose

Rubus aboriginum Rydb. garden dewberry

Rubus allegheniensis Porter Allegheny blackberry

Rubus frondosus Bigelow yankee blackberry

Rubus idaeus L. ssp. strigosus (Michx.) Focke grayleaf red raspberry

Rubus ostryafolius Rydb. highbush blackberry

Rubus trivialis Michx. southern dewberry

Sanguisorba annua (Nutt. ex Hook.) Torr. \& Gray

prairie burnet
Rubiaceae

Cephalanthus occidentalis L. common buttonbush

Diodia teres Walt. poorjoe

Galium aparine L. stickywilly

Galium circaezans Michx. var. hypomalacum Fern. licorice bedstraw

Galium obtusum Bigelow bluntleaf bedstraw

Galium pilosum Ait. hairy bedstraw

Galium pilosum Ait. var. pilosum hairy bedstraw

Galium texense Gray Texas bedstraw

Galium virgatum Nutt. southwestern bedstraw

Hedyotis nigricans (Lam.) Fosberg diamondflowers

Hedyotis nigricans (Lam.) Fosberg var. nigricans diamondflowers

Houstonia longifolia var. longifolia Gaertn.

Houstonia purpurea L. var. purpurea Venus' pride Houstonia pusilla Schoepf tiny bluet

Sherardia arvensis L. blue fieldmadder

\section{Rutaceae}

Ptelea trifoliata L. common hoptree

Ptelea trifoliata L. ssp. polyadenia (Greene) V. Bailey pallid hoptree

Ptelea trifoliata L. ssp. trifoliata var. trifoliate common hoptree 
Ptelea trifoliata ssp. trifoliata L. var. mollis Torr. \& Gray common hoptree

\section{Salicaceae}

Populus deltoides Bartr. ex Marsh. eastern cottonwood

Salix caroliniana Michx. coastal plain willow

Salix nigra Marsh. black willow

\section{Santalaceae}

Comandra umbellata (L.) Nutt. ssp. pallida (A. DC.) Piehl pale bastard toadflax

\section{Sapindaceae}

Cardiospermum halicacabum L. love in a puff

Sapindus drummondii Hook. \& Arn. Sapindus saponaria L.

var. drummondii (Hook. \& Arn.) L.Benson western soapberry

\section{Sapotaceae}

Sideroxylon lanuginosum Michx. gum bully

Sideroxylon lanuginosum Michx. ssp. rigidum (Gray)

T.D. Pennington

\section{Scrophulariaceae}

Agalinis heterophylla (Nutt.)

Small ex Britt.

prairie false foxglove

Bacopa rotundifolia (Michx.) Wettst. disk waterhyssop

Castilleja coccinea (L.) Spreng. scarlet Indian paintbrush

Castilleja purpurea (Nutt.) G. Don downy Indian paintbrush

Castilleja purpurea (Nutt.) G. Don var. citrina (Pennell) Shinners prairie Indian paintbrush
Collinsia verna Nutt. spring blue eyed Mary

Collinsia violacea Nutt. violet blue eyed Mary

Gratiola sphaerocarpa Ell.

Gratiola virginiana L. roundfruit hedgehyssop

Leucospora multifida (Michx.) Nutt. narrowleaf paleseed

Lindernia anagallidea (Michx.) Pennell

Lindernia dubia (L.) Pennell yellowseed false pimpernel

Macuillamia rotundifolia (Michx.) Raf.

Mimulus glabratus Kunth roundleaf monkeyflower

Mimulus glabratus Kunth var. jamesii (Torr. \& Gray ex Benth.) Gray James' monkeyflower

Nuttallanthus canadensis (L.)

D.A. Sutton

Canada toadflax

Nuttallanthus texanus (Scheele)

D.A. Sutton

Texas toadflax

Penstemon albidus Nutt. white penstemon

Penstemon australis Small ssp. laxiflorus (Pennell) Bennett

Penstemon cobaea Nutt. cobaea beardtongue

Penstemon fendleri Torr. \& Gray Fendler's penstemon

Penstemon oklahomensis Pennell Oklahoma beardtongue

Scrophularia lanceolata Pursh lanceleaf figwort

Veronica arvensis L. corn speedwell

Veronica peregrina L. neckweed

Veronica peregrina L. ssp. xalapensis (Kunth) Pennell hairy purslane speedwell 
Simaroubaceae

Ailanthus altissima (P. Mill.) Swingle tree of heaven

\section{Smilacaceae}

Smilax bona-nox L. saw greenbrier

Smilax rotundifolia L. roundleaf greenbrier

Smilax tamnoides $\mathrm{L}$. bristly greenbrier

\section{Solanaceae}

Datura meteloides auct. p.p. non Dunal Physalis angulata L. cutleaf groundcherry

Physalis cinerascens (Dunal) A.S. Hitchc. var. cinerascens smallflower groundcherry

Physalis heterophylla Nees clammy groundcherry

Physalis lobata Torr.

Physalis longifolia Nutt. longleaf groundcherry

Physalis mollis Nutt. field groundcherry

Physalis mollis Nutt. var. mollis field groundcherry Physalis pubescens L. var. integrifolia (Dunal) Waterfall husk tomato

Physalis pumila Nutt. dwarf groundcherry

Physalis viscosa L. ssp. mollis (Nutt.) Waterfall Quincula lobata (Torr.) Raf. Chinese lantern

Solanum citrullifolium A. Braun watermelon nightshade

Solanum dimidiatum Raf. western horsenettle

Solanum elaeagnifolium Cav. silverleaf nightshade
Solanum nigrum auct. non L.

Solanum ptychanthum Dunal West Indian nightshade

Solanum rostratum Dunal buffalobur nightshade

Tamaricaceae

Tamarix gallica L. salt cedar

\section{Ulmaceae}

Celtis laevigata Willd. sugarberry

Celtis laevigata Willd. var. reticulata (Torr.) L. Benson netleaf hackberry

Celtis laevigata Willd. var. texana Sarg.

Texan sugarberry

Ulmus americana L.

American elm

Ulmus rubra Muhl. slippery elm

\section{Urticaceae}

Boehmeria cylindrica (L.) Sw. smallspike false nettle

Boehmeria cylindrica (L.) Sw. var. drummondiana (Weddell) Weddell

Parietaria pensylvanica Muhl. ex Willd. Pennsylvania pellitory

Parietaria pensylvanica Muhl. ex Willd. var. obtusa (Rydb. ex Small) Shinners

Valerianaceae

Valerianella radiata (L.) Dufr. beaked cornsalad

\section{Verbenaceae}

Glandularia bipinnatifida (Nutt.) Nutt. var. bipinnatifida

Dakota mock vervain

Glandularia canadensis (L.) Nutt.

rose mock vervain 
Glandularia pumila (Rydb.) Umber pink mock vervain

Lippia lanceolata Michx.

Phryma leptostachya L. American lopseed

Phyla incisa Small

Phyla lanceolata (Michx.) Greene lanceleaf fogfruit

Verbena bipinnatifida Nutt.

Verbena bracteata Lag. \& Rodr. bigbract verbena

Verbena canadensis (L.) Britt.

Verbena pumila Rydb.

Verbena stricta Vent. hoary verbena

Verbena urticifolia L. white vervain

\section{Violaceae}

Hybanthus verticillatus (Ortega)

Baill. babyslippers

Viola bicolor Pursh field pansy

Viola missouriensis Greene

Viola retusa Greene

Viola sororia Willd. common blue violet

\section{Viscaceae}

Phoradendron flavescens Nutt. ex Engelm.

Phoradendron leucarpum (Raf.)

Reveal \& M.C. Johnston

oak mistletoe

\section{Vitaceae}

Ampelopsis cordata Michx. heartleaf peppervine

Cissus incisa auct. non Des Moulins

Cissus trifoliata (L.) L. sorrelvine

Parthenocissus quinquefolia (L.) Planch. Virginia creeper

Parthenocissus vitacea (Knerr) A.S. Hitchc. woodbine

Vitis cinerea (Engelm.) Millard graybark grape

Vitis cinerea (Engelm.) Millard var. cinerea graybark grape

Vitis riparia Michx. riverbank grape

Vitis rupestris Scheele sand grape

Vitis vulpina $\mathrm{L}$. frost grape

\section{Zygophyllaceae}

Tribulus terrestris L.

Puncturevine 